\title{
Autologous cytokine-induced killer cell transfusion increases overall survival in advanced pancreatic cancer
}

\author{
Zibing Wang ${ }^{1}$, Yuqing Liu², Rui'e Li', Yiman Shang ${ }^{1}$, Yong Zhang ${ }^{1}$, Lingdi Zhao' ${ }^{1}$, Wei Li', Yonghao Yang ${ }^{1}$, \\ Xiaojie Zhang ${ }^{1}$, Tiejun Yang ${ }^{3}$, Changfu Nie ${ }^{4}$, Feng Han ${ }^{4}$, Ying Liu ${ }^{5}$, Suxia Luo ${ }^{5}$, Quanli Gao ${ }^{1 *}$ and Yongping Song ${ }^{6 *}$
}

\begin{abstract}
Background: Advanced pancreatic cancer (PC) has very poor prognosis with present treatments, thus necessitating continued efforts to find improved therapeutic approaches. Both preclinical and preliminary clinical data indicate that cytokine-induced killer (CIK) cells are an effective tool against various types of solid tumors. Here, we conducted a study to determine whether CIK cell-based therapy (CBT) can improve the outcomes of advanced PC.

Methods: Eighty-two patients with advanced PC, whose predicted survival time was longer than 3 months, were analyzed retrospectively. Of all the patients, 57 individuals were receiving chemotherapy, while the remaining 25 individuals were treated with CBT.

Results: The overall survival analysis was based on 48 deaths in the 57 patients in the chemotherapy group (84.2\%) and 18 deaths in the 25 patients in the CBT group (72.0\%). In the CBT group, the median overall survival time was 13.5 months, as compared to 6.6 months in the chemotherapy group (hazard ratio for death, 0.39; $95 \%$ confidence interval, 0.23 to $0.65 ; p<0.001$ ). The survival rate was $88.9 \%$ in the CBT group versus $54.2 \%$ in the chemotherapy group at 6 months, $61.1 \%$ versus $12.5 \%$ at 12 months, and $38.9 \%$ versus $4.2 \%$ at 18 months. The disease control rate was $68.0 \%$ in the CBT group and $29.8 \%$ in the chemotherapy group $(p<0.001)$.

Conclusions: These results from this retrospective analysis appeared to imply that CBT might prolong survival in these high-risk PC patients. Prospective study is needed to corroborate this observation.
\end{abstract}

Keywords: Cytokine-induced killer cells, Immunotherapy, Pancreatic cancer, Overall survival

\section{Background}

Pancreatic cancer (PC) has the poorest prognosis among all gastrointestinal cancers, with 1-year survival rate of around $20 \%$ and 5-year survival rate of $7 \%$ for diagnosed patients [1-3]. Gemcitabine is a chemical agent used as standard chemotherapy treatment for advanced PC. However, patients treated with this agent alone have a median overall survival time (mOS) of no more than 8.3 months, and the results of most clinical trials show that the mOS of advanced PC patients is not significantly prolonged

\footnotetext{
* Correspondence: quanligao1@aliyun.com; songyongping@medmail.com.cn Zibing Wang and Yuqing Liu share co-first authorship.

'Department of Immunotherapy, Affiliated Cancer Hospital of Zhengzhou

University and Henan Cancer Hospital, Zhengzhou 450008, China

${ }^{6}$ Department of Hematology, Affiliated Cancer Hospital of Zhengzhou

University and Henan Cancer Hospital, Zhengzhou 450008, China

Full list of author information is available at the end of the article
}

when gemcitabine is combined with other cytotoxic or targeted agents [3-5]. Patients who received FOLFIRINOX (oxaliplatin, irinotecan, fluorouracil, and leucovorin) or nab-paclitaxel combined with gemcitabine therapy show an improvement in mOS, with an increase of 4.3 or 1.8 months, respectively [2, 6]. However, these strategies are associated with a higher incidence of serious side effects, and the patients must therefore undergo rigorous testing prior to chemotherapy to have evaluated suitability for therapy, and further require close monitoring during the therapy. Thus, new therapeutic strategies for advanced PC treatment are urgently needed.

A promising approach treating PC is the use of immune checkpoint inhibitors. The targets of these treatments are the molecules that serve as checks in the regulation of immune responses. By blocking inhibitory 
molecules, these treatments will activate the immune system and as such reactivate preexisting anti-cancer responses [7-11]. Several checkpoint inhibitors are currently in development.

Another major avenue of immunotherapy for PC is adoptive $\mathrm{T}$ cell transfer. Cytokine-induced killer (CIK) cells are a heterogeneous subset of $\mathrm{T}$ lymphocytes expanded ex vivo that express CD3 and CD56 as well as natural killer group 2, member D molecules (NKG2D). Adoptive transfer of CIK cells has been shown to be effective for cancer treatment, with high safety, as indicated by the prolonged survival of patients with different types of tumors [12-17]. When used in combination with chemotherapy, CIK cells show enhanced efficacy in preventing disease recurrence and improving the prognosis of cancer patients [18-20]. Recently, researchers have been taking advantage of CIK cells as a second-line treatment to treat advanced PC, and the results obtained showed encouraging results, both in terms of single use and combined use with other treatments. For example, in a phase 2 clinical trial, patients with CIK cells in gemcitabine-refractory advanced PC displayed a mOS of 6.2 months [21]. Another clinical study showed that patients who received CIK cells in combination with S-1, an oral fluoropyrimidine derivative, in gemcitabinerefractory advanced PC displayed a mOS of 6.6 months, which is longer than mOS of patients receiving S-1 alone (6.1 months) [22]. However, data are lacking on the efficacy of CIK cells as a first-line treatment in patients with advanced PC.

In this study, we performed a retrospective study, where we compared the mOS of patients with advanced $\mathrm{PC}$, who have been treated with $\mathrm{CBT}$ or chemotherapy as a first-line treatment.

\section{Results}

\section{Characteristics of the patients}

A total number of 82 patients were enrolled in this study. Of these, 57 patients received chemotherapy alone, and 25 patients received CBT. The last follow-up examination of the patients was performed on September 13, 2014. Although sample numbers were different in the two groups, the baseline demographic and clinical characteristics of patients were relatively well balanced (Table 1 and Additional files 1 and 2).

\section{Survival}

The overall survival analysis was based on 48 deaths in the 57 patients in the chemotherapy group $(84.2 \%)$ and 18 deaths in the 25 patients in the CBT group (72.0\%). The mOS was 13.5 months in the CBT, as compared with 6.6 months in the chemotherapy group (hazard ratio for death, $0.39 ; 95 \% \mathrm{CI}, 0.23$ to $0.65 ; p<0.001$ ) (Fig. 1). The overall survival rates at 6,12 , and 18 months were 88.9, 61.1, and $38.9 \%$, respectively, in the CBT group, as compared with the survival rate of 54.2, 12.5, and $4.2 \%$ in the chemotherapy group.

\section{Response}

Of 57 patients in the chemotherapy group, no complete responses were seen, 3 patients exhibited a partial response (5.3\%), 14 patients displayed stable disease (24.6\%), and 40 patients showed progressive disease (70.2 \%) (Fig. 2a). Of 25 patients in the CBT group, 1 complete response was seen (4.0\%), 2 patients exhibited a partial response $(8.0 \%), 14$ patients displayed stable disease $(56.0 \%)$, and 8 patients showed progressive disease (32.0\%) (Fig. 2b). The DCR rate was $29.8 \%(17 / 57)$ in the chemotherapy group and $68.0 \%(17 / 25)$ in the CBT group. The DCR rate was significantly higher in the CBT group than in the chemotherapy group. $(p<0.001$, Fig. 3).

\section{Adverse events}

The major adverse events are listed in Table 2. Patients in the CBT group seemed to have higher incidences of fever and fatigue as compared with patients in the chemotherapy group. The difference was minimal, statistically not significant, and was considered to be attributed to the most common side effect of IL-2 administrated after CIK cell transfusion [14]. There were no significant differences in leucopenia, neutropenia, thrombocytopenia, nausea, diarrhea, vomiting, and infection between the two groups.

\section{Discussion}

Overall survival time has traditionally been regarded as the most reliable endpoint in evaluating an experimental strategy for cancer treatment, and thus its improvement is an important criterion for regulatory approval of a new therapy. However, most large-scale clinical phase 3 studies have not shown significantly improved survival in advanced PC. Our data presented here suggest that in patients with advanced PC, the overall survival was significantly improved when the patients were treated with CBT, relative to patients who received chemotherapy alone. More importantly, CIK cell-associated toxicity was mild, with fever as the main side effect, at an incidence rate of approximately $15 \%$ [14]. This suggests that CIK cell treatment has a higher efficacy and safety in advanced PC.

Gemcitabine and fluorouracil are two widely used chemotherapeutical agents in advanced PC. Previous studies have shown that gemcitabine suppresses the production of IgG antibody but does not affect the specific antitumor immunity [23, 24]. In another study, it was shown that the anti-tumor effect of gemcitabine does not result from direct cytotoxic effects on tumor cells but rather from an enhancement of $\mathrm{T}$ cell-mediated immune responses [25]. These data suggest that gemcitabine acts 
Table 1 Demographic and clinicopathological characteristics of the patients at baseline

\begin{tabular}{|c|c|c|c|c|c|}
\hline \multirow[t]{2}{*}{ Characteristics } & \multicolumn{2}{|c|}{ Chemotherapy $(n=57)$} & \multicolumn{2}{|c|}{$\mathrm{CBT}(n=25)$} & \multirow[t]{2}{*}{$p$ value } \\
\hline & Number & $\%$ & Number & $\%$ & \\
\hline \multicolumn{6}{|l|}{ Sex } \\
\hline Male & 35 & 61.40 & 15 & 60.00 & \multirow[t]{2}{*}{1.00} \\
\hline Female & 22 & 38.60 & 10 & 40.00 & \\
\hline \multicolumn{6}{|l|}{ Age, years } \\
\hline$<65$ & 38 & 66.67 & 14 & 56.00 & \multirow[t]{2}{*}{0.46} \\
\hline$\geq 65$ & 19 & 33.33 & 11 & 44.00 & \\
\hline \multicolumn{6}{|l|}{ Diagnosis basis } \\
\hline Pathologically & 29 & 50.88 & 14 & 56.00 & \multirow[t]{2}{*}{0.81} \\
\hline Clinically & 28 & 49.12 & 11 & 44.00 & \\
\hline \multicolumn{6}{|l|}{ Extent of disease } \\
\hline Locally advanced & 9 & 15.79 & 2 & 8.00 & \multirow[t]{2}{*}{0.49} \\
\hline Metastatic & 48 & 84.21 & 23 & 92.00 & \\
\hline \multicolumn{6}{|c|}{ Measurable metastatic sites } \\
\hline Liver $^{\mathrm{a}}$ & 30 & 52.63 & 13 & 52.00 & \multirow[t]{2}{*}{1.00} \\
\hline Other & 27 & 47.37 & 12 & 48.00 & \\
\hline \multicolumn{6}{|l|}{ No. of metastatic sites } \\
\hline 1 & 32 & 56.14 & 16 & 64.00 & \multirow[t]{3}{*}{0.83} \\
\hline 2 & 18 & 31.58 & 7 & 28.88 & \\
\hline$\geq 3$ & 7 & 12.28 & 2 & 8.00 & \\
\hline \multicolumn{6}{|c|}{ ECOG performance status score } \\
\hline 1 & 20 & 35.09 & 9 & 36.00 & \multirow[t]{2}{*}{1.00} \\
\hline 2 & 37 & 64.91 & 16 & 64.00 & \\
\hline \multicolumn{6}{|c|}{ Pancreatic tumor location } \\
\hline Head & 21 & 36.84 & 14 & 56.00 & \multirow[t]{4}{*}{0.17} \\
\hline Body & 5 & 8.78 & 4 & 16.00 & \\
\hline Tail & 16 & 28.07 & 3 & 12.00 & \\
\hline Multicentric $^{b}$ & 15 & 26.32 & 4 & 16.00 & \\
\hline \multicolumn{6}{|c|}{ Level of carbohydrate antigen 19-9- u/ml } \\
\hline Abnormal & 47 & 82.46 & 20 & 80.00 & \multirow[t]{3}{*}{0.23} \\
\hline Normal & 6 & 10.53 & 5 & 20.00 & \\
\hline Unknown ${ }^{c}$ & 4 & 7.02 & 0 & 0.00 & \\
\hline
\end{tabular}

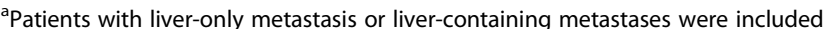

${ }^{\mathrm{b}}$ When tumor infiltrated more than one region of pancreas, it was defined as multicentric. It included head-body, head-tail, body-tail, and head-body-tail tumors

${ }^{c}$ Four patients did not carry out this detection for economic condition

as an immunomodulator, making it a good candidate drug for combination with immunotherapy strategies. This notion is supported by two experimental studies showing that combination of a dendritic cell vaccine with gemcitabine improved the survival of tumorbearing hosts in a murine pancreatic carcinoma model $[26,27]$. Fluorouracil also exhibited immunomodulatory effects when combined with immunotherapy strategies [28]. Thus, the immunomodulatory effects of gemcitabine and fluorouracil play an important role for hosts to elicit robust anti-tumor immunity. On the basis of these data and our results, we propose that when combined with immunomodulatory chemotherapeutical agents, CIK cells exhibit even higher, more potent anti-tumor activity.

Previous in vitro experiments show that cultured CIK cells possess significant cytotoxic activity against tumor cells and most of the cytotoxicity is attributed to the higher proliferation of $\mathrm{CD}^{+} \mathrm{CD}^{2} 6^{+}$cells $[29,30]$. In vivo, CIK cells can migrate to the site of tumors through interacting with chemokine receptors expressed on the surface of them, where they release their cytotoxic 


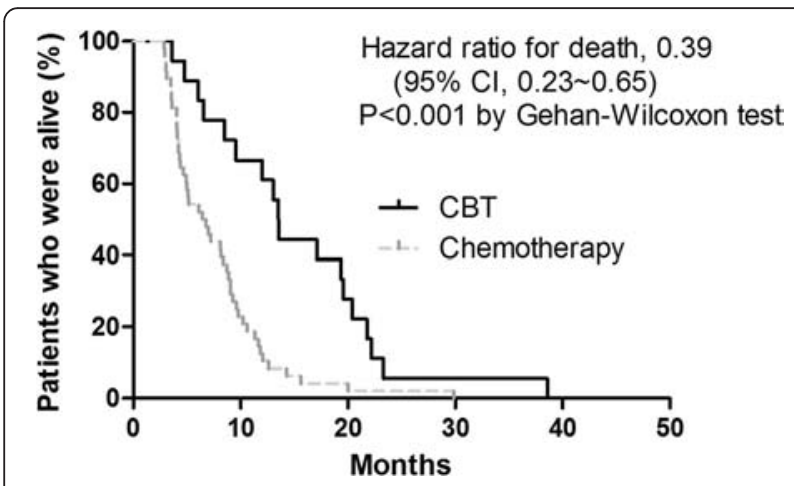

Fig. 1 Overall survival time for CBT versus chemotherapy alone in patients with advanced PC. Overall survival was calculated in 18 patients with CBT compared with 48 patients with chemotherapy

potential and inhibit tumor growth [31-33]. The molecular mechanisms of tumor recognition and killing by CIK cells possibly involve the expression of lymphocyte function-associated antigens, NKG2D, DNAS accessory molecule-1, and NKp30 [29, 34]. Besides their direct cytotoxic effect, CIK cells secret interferon gamma that modulates the expression of adhesion molecules on tumor cells, and the altered expression pattern of adhesion molecule enhances apoptosis that is induced by

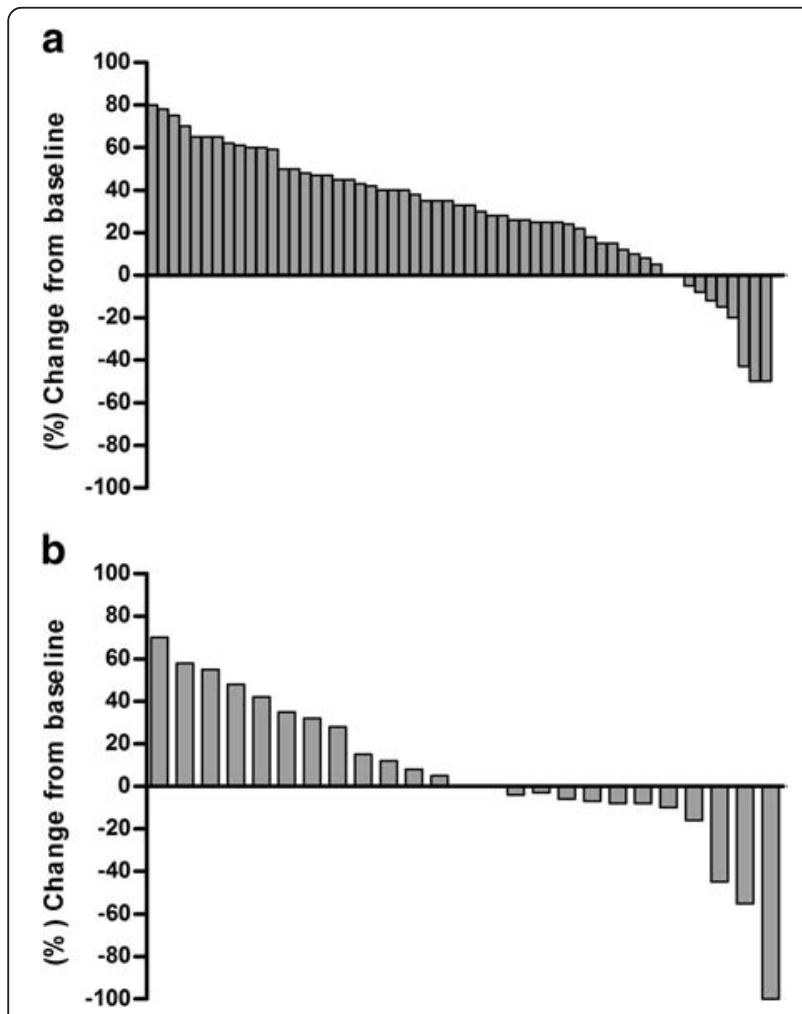

Fig. 2 Waterfall chart demonstrating changes in tumor measurement with measurable tumor at baseline. Response to therapy was calculated in 57 patients with chemotherapy (a) compared with 25 patients with CBT (b)

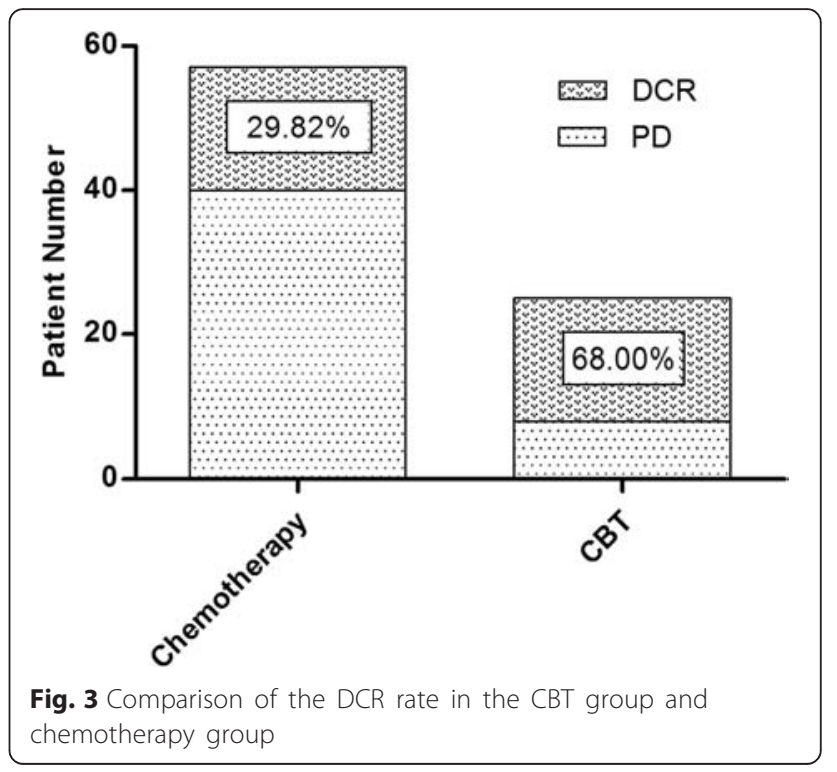

cytotoxic effector cells [35]. In addition, recent studies report that CIK cells attack cancer stem cells in both animal models and human patients [13,33]. Finally, previous work using a mouse model indicates that cancer immunotherapy using interferon alpha can significantly reduce chemotherapy-induced expression of multi-drug resistance proteins, as well as lower the activity of drug efflux out of the cancer cells subjected to this treatment, thus rescuing their chemosensitivity [36]. This result suggests that CIK cells increase the sensitivity of PC cells to gemcitabine and/or S-1, which is another possible mechanism for CIK cell anti-tumor action.

Recently, Arina and colleagues suggest that the presence of myeloid-derived suppressor cells (MDSC) limits adoptively transferred $\mathrm{T}$ cell infiltration and function, and manipulation of intratumoral myeloid cells may improve the outcome of otherwise unsuccessful adoptive transferred T cells [37]. Both gemcitabine and fluorouracil were known to be selectively cytotoxic on MDSC whereas no significant effect on T cells in animal models $[38,39]$, and MDSC decrease was observed when these chemotherapeutic agents were used in cancer patients $[40,41]$. Thus, it is reasonable that CIK cells combined with these agents led to a better effect in advanced PC patients.

Our survival analysis has several major experimental limitations. First of all, this is a retrospective, nonrandomized study, and only patients from medical wards of our hospital were included, which may not truly reflect patients from other hospitals. Moreover, survival differences between CBT and chemotherapy might be affected by differences during patient selection and differences in standard and supportive care treatment. A second limitation of our analysis is that we did not have 
Table 2 Adverse events (AEs) among patients in chemotherapy and CBT groups

\begin{tabular}{|c|c|c|c|c|c|}
\hline \multirow[t]{2}{*}{ Adverse event } & \multicolumn{2}{|c|}{ Chemotherapy $(n=57)$} & \multicolumn{2}{|c|}{ CBT $(n=25)$} & \multirow[t]{2}{*}{$p$ value } \\
\hline & Number & $\%$ & Number & $\%$ & \\
\hline Myelosuppression $^{a}$ & 33 & 57.89 & 10 & 40.00 & 0.16 \\
\hline Digestive tract symptom ${ }^{b}$ & 18 & 31.58 & 5 & 20.00 & 0.42 \\
\hline Fever & 2 & 3.51 & 3 & 12.00 & 0.16 \\
\hline Fatigue & 2 & 3.51 & 2 & 8.00 & 0.58 \\
\hline Infection & 2 & 3.51 & 0 & 0 & 1.00 \\
\hline
\end{tabular}

${ }^{a}$ Myelosuppression includes thrombocytopenia, lymphopenia, and neutropenia

${ }^{\mathrm{b}}$ Digestive tract symptom includes nausea, vomiting, and diarrhea

detailed information on the post-chemotherapy treatment in the chemotherapy group, and this could contribute to the difference in mOS between the two groups. Thirdly, the use of chemotherapeutic agents in the two groups was not standardized. Despite this difference in chemical agents between the two groups, we believe that such difference had no significant influence on the difference in mOS, since the results of previous clinical trials clearly indicated that there was no significant increase in $\mathrm{mOS}$ when two or more drugs were combined. Thus, the observed therapeutic benefits in the combined group were considered to be a result of the transfusion with CIK cells.

\section{Conclusions}

In summary, the fact that we observed prolonged survival for patients receiving CBT supports the notion that CIK cells can greatly improve the prognosis of advanced PC. Based on the data presented here, other estimates of clinical benefit such as objective response rate and progression-free survival and improved quality of life, CBT will hopefully become a standard of care for patients with advanced PC.

\section{Methods}

\section{Patient selection}

Patients in this retrospective analysis were admitted between 1 September 2010 and 4 May 2014 in Henan Cancer Hospital. The criteria for patients included in the study were the following: (1) PC was histologically or cytologically confirmed (pathologically diagnosed), or diagnosed by symptoms and complications, computer tomography or magnetic resonance imaging, combined with measurement of serum carbohydrate antigen 199 levels (clinically diagnosed). (2) The ECOG performance status was below 3. (3) The predicted survival time was longer than 3 months. (4) Age is larger than 18 years. (5) Uncontrolled infection was absent. Unresectability was assessed by an experienced surgeon either during laparotomy or by radiologic work-up (computed tomography scan and/or magnetic resonance imaging) showing portal and/or mesenteric and/or celiac vascular involvement. Any extrapancreatic disease including Vater's ampulloma and adenocarcinoma of the biliary tract was an exclusion criterion. We listed all patients who met the above criteria and surveyed their records. All patients gave written informed consent, which had been approved by the institutional review board of Zhengzhou University. This study was conducted in accordance with the provisions of the Declaration of Helsinki and Good Clinical Practice guidelines.

\section{Study design}

CIK cells were prepared according to the procedures described in a previous study [13]. Briefly, peripheral blood mononuclear cell were separated and cultured under sterile conditions with 1640 medium containing anti-CD3 monoclonal antibody, interferon gamma, interleukin-2, and RetroNectin (RN, Takara, Japan). After culturing of cells for 10 to 14 days, a target dose of about $5 \times 10^{9} \mathrm{CIK}$ cells with over $95 \%$ viability was obtained and tested for biological contaminants. The modified method led to a significant higher proportion of $\mathrm{CD}^{+} \mathrm{CD}^{+} 6^{+}$cellular subset [14]. Anti-tumor cytokines such as tumor necrosis factor alpha were higher and pro-tumor cytokines such as interleukin-4 and interleukin-5 were lower in the CIK cell cultures prepared with the modified method (data not shown). Cells were then prepared in $2 \%$ albumin containing sodium chloride solution before transfusion into the patients. In the following 3 days, patients were administered with interleukin-2 (2 million IU per day) to promote CIK activity. Patients in this study received at least 2 cycles of CIK cell transfusion.

In the CBT group, 21 patients received CIK cell transfusion plus gemcitabine $\left(1000 \mathrm{mg} / \mathrm{m}^{2}, \mathrm{~d} 1,8\right)$ and/or S-1 (orally twice daily at a dose according to the body surface area $\left(<1.25 \mathrm{~m}^{2}, 80 \mathrm{mg} /\right.$ day; $1.25-1.5 \mathrm{~m}^{2}, 100 \mathrm{mg} /$ day; $>1.5 \mathrm{~m}^{2}, 120 \mathrm{mg} /$ day) on days 1-14), one patient received CIK cell transfusion plus epotoside $\left(60 \mathrm{mg} / \mathrm{m}^{2}, \mathrm{~d} 1-5\right)$, and three cases received only CIK cells. Transfusion of CIK cells was followed at the end of each cycle of chemotherapy. Patients received chemotherapy every 3-week period.

In the chemotherapy group, 54 patients received gemcitabine $\left(1000 \mathrm{mg} / \mathrm{m}^{2}, \mathrm{~d} 1,8\right)$ and/or S-1-based (orally twice daily at a dose according to the body surface area 
$\left(<1.25 \mathrm{~m}^{2}, 80 \mathrm{mg} /\right.$ day; $1.25-1.5 \mathrm{~m}^{2}, 100 \mathrm{mg} /$ day; $>1.5 \mathrm{~m}^{2}$, $120 \mathrm{mg} /$ day) on days 1-14) chemotherapy, one patient received epotoside $\left(60 \mathrm{mg} / \mathrm{m}^{2}, \mathrm{~d} 1-5\right)$ and cisplatin $(15 \mathrm{mg} /$ $\left.\mathrm{m}^{2}, \mathrm{~d} 2-5\right)$, one patient received nab-paclitaxel $(110 \mathrm{mg} /$ $\left.\mathrm{m}^{2}, \mathrm{~d} 1,8\right)$ and gemcitabine $\left(1000 \mathrm{mg} / \mathrm{m}^{2}, \mathrm{~d} 1,8\right)$, and one patient received an intraperitoneal perfusion of cisplatin $\left(20 \mathrm{mg} / \mathrm{m}^{2}, \mathrm{~d} 1,2\right)$. Patients received chemotherapy every 3-week period.

\section{Evaluation of short-term efficacy and toxicity}

The tumor response was assessed according to the Response Evaluation Criteria in Solid Tumors (RECIST): complete response (CR), partial response (PR), stable disease (SD), and progressive disease (PD). We performed tumor assessments with the use of computed tomographic (CT) scanning at baseline and 2 months post treatment start. Efficacy was evaluated by disease control rate (DCR), consisting of CR, PR, and SD. Safety was assessed by documentation of adverse events. Hematologic and serum chemical measurement were performed before and after each cycle of treatment. Adverse events were graded with the use of the Common Terminology Criteria for Adverse Events of the National Cancer Institute, version 3.0.

\section{Statistical analysis}

Fisher's exact test or chi-square test were used to assess the association of the treatment group with demographic and clinicopathological characteristics as well as the response to therapy. Overall survival was estimated using the Kaplan-Meier method, and the log-rank test was used to compare the difference between the two groups. The hazard ratio between the two groups was estimated by proportional hazards regression using a $95 \%$ Wald confidence interval $(95 \% \mathrm{CI})$. Data analysis was done with Graphpad Prism 5, with all $p$ values applied as twosided.

\section{Additional files}

Additional file 1: Demographic and clinical characteristics of individual patient in chemotherapy group. (DOC $85 \mathrm{~kb}$ )

Additional file 2: Demographic and clinical characteristics of individual patient in CBT group. (DOC $43 \mathrm{~kb}$ )

\section{Abbreviations}

CBT: cytokine-induced killer cell-based therapy; CIK: cytokine-induced killer; DCR: disease control rate; MDSC: myeloid-derived suppressor cells; mOS: median overall survival time; PC: pancreatic cancer.

\section{Competing interests}

The authors declare that they have no competing interests.

\section{Authors' contributions}

ZW, QG, and YS designed the study and analyzed the data. YL, RL, YS, YZ, LZ, $W L, Y Y$, and $X Z$ collected data. $Z W$ wrote the report. TY, CN, FH, YL, and SL interpreted the data. All authors read and provided comments on the report. All authors read and approved the final manuscript.

\section{Acknowledgements}

This work was supported by the National Natural Science Foundation of China (81000914) and Foundation of Henan Health Committee (2011010011). The authors thank all our patients who participated in this study, together with their families. The authors thank Dr. Torsten Juelich for linguistic assistance during the preparation of this manuscript.

\section{Author details}

'Department of Immunotherapy, Affiliated Cancer Hospital of Zhengzhou University and Henan Cancer Hospital, Zhengzhou 450008, China. ${ }^{2}$ Department of Oncology, Third Affiliated Hospital of Xinxiang Medical College, Xinxiang 453003, China. ${ }^{3}$ Department of Urology, Affiliated Cancer Hospital of Zhengzhou University and Henan Cancer Hospital, Zhengzhou 450008, China. ${ }^{4}$ Department of Hepatobiliary and Pancreatic Surgery, Affiliated Cancer Hospital of Zhengzhou University and Henan Cancer Hospital, Zhengzhou 450008, China. ${ }^{5}$ Department of Oncology, Affiliated Cancer Hospital of Zhengzhou University and Henan Cancer Hospital, Zhengzhou 450008, China. ${ }^{6}$ Department of Hematology, Affiliated Cancer Hospital of Zhengzhou University and Henan Cancer Hospital, Zhengzhou 450008, China.

\section{Received: 3 November 2015 Accepted: 24 January 2016}

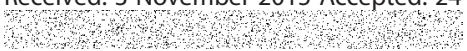

\section{References}

1. Ko AH. Progress in the treatment of metastatic pancreatic cancer and the search for next opportunities. J Clin Oncol. 2015;33:1779-86.

2. Conroy T, Desseigne F, Ychou M, Bouché O, Guimbaud R, Bécouarn Y, et al. FOLFIRINOX versus gemcitabine for metastatic pancreatic cancer. $N$ Engl J Med. 2011;364:1817-25.

3. Hu J, Zhao G, Wang H, Tang L, Xu Y, Ma Y, et al. A meta-analysis of gemcitabine containing chemotherapy for locally advanced and metastatic pancreatic adenocarcinoma. J Hematol Oncol. 2011;4:1.

4. Bernardo HL G, Clark JW, Lauwers GY, Ryan DP, Nina G, Alona M, et al. Long term survivors with metastatic pancreatic adenocarcinoma treated with gemcitabine: a retrospective analysis. J Hematol Oncol. 2009;2:13.

5. Colucci G, Labianca R, Di Costanzo F, Gebbia V, Cartenì G, Massidda B, et al. Randomized phase III trial of gemcitabine plus cisplatin compared with single-agent gemcitabine as first-line treatment of patients with advanced pancreatic cancer: the GIP-1 study. J Clin Oncol. 2010;28:1645-51.

6. Von Hoff DD, Ervin T, Arena FP, Chiorean EG, Infante J, Moore M, et al. Increased survival in pancreatic cancer with nab-paclitaxel plus gemcitabine. N Engl J Med. 2013;369:1691-703.

7. Goel G, Sun W. Advances in the management of gastrointestinal cancers-an upcoming role of immune checkpoint blockade. J Hematol Oncol. 2015:8:86.

8. Goel G, Sun W. Novel approaches in the management of pancreatic ductal adenocarcinoma: potential promises for the future. J Hematol Oncol. 2015:8:44.

9. Lin A, Lin E. Programmed death 1 blockade, an Achilles heel for MMRdeficient tumors? J Hematol Oncol. 2015;8:124.

10. Akinleye A, Iragavarapu C, Furqan M, Cang S, Liu D. Novel agents for advanced pancreatic cancer. Oncotarget. 2015;6:39521-37.

11. Tsai K, Daud A. Nivolumab plus ipilimumab in the treatment of advanced melanoma. J Hematol Oncol. 2015;8:123.

12. Takayama T, Sekine T, Makuuchi M, Yamasaki S, Kosuge T, Yamamoto J, et al. Adoptive immunotherapy to lower postsurgical recurrence rates of hepatocellular carcinoma: a randomised trial. Lancet. 2000;356:802-7.

13. Gammaitoni L, Giraudo L, Leuci V, Todorovic M, Mesiano G, Picciotto F, et al. Effective activity of cytokine-induced killer cells against autologous metastatic melanoma including cells with stemness features. Clin Cancer Res. 2013;19:4347-58.

14. Wang Z, Zhang Y, Liu Y, Wang L, Zhao L, Yang T, et al. Association of myeloid-derived suppressor cells and efficacy of cytokine-induced killer cell immunotherapy in metastatic renal cell carcinoma patients. J Immunother. 2014;37:43-50.

15. Schmeel L, Schmeel F, Coch C, Schmidt-Wolf IG. Cytokine-induced killer (CIK) cells in cancer immunotherapy: report of the international registry on CIK cells (IRCC). J Cancer Res Clin Oncol. 2015;141:839-49.

16. Schmidt-Wolf IG, Negrin RS, Kiem HP, Blume KG, Weissman IL. Use of a SCID mouse/human lymphoma model to evaluate cytokine-induced killer cells with potent antitumor cell activity. J Exp Med. 1991;174:139-49. 
17. Wang Y, Xu Z, Zhou F, Sun Y, Chen J, Li L, et al. The combination of dendritic cells-cytotoxic T Iymphocytes/cytokine-induced killer (DC-CTL/CIK) therapy exerts immune and clinical responses in patients with malignant tumors. Exp Hematol Oncol. 2015;4:32.

18. Wu C, Jiang J, Shi L, Xu N. Prospective study of chemotherapy in combination with cytokine-induced killer cells in patients suffering from advanced non-small cell lung cancer. Anticancer Res. 2008;28:3997-4002.

19. Li J, Gu M, Pan K, Liu L, Zhang H, Shen W, et al. Autologous cytokineinduced killer cell transfusion in combination with gemcitabine plus cisplatin regimen chemotherapy for metastatic nasopharyngeal carcinoma. J Immunother. 2012;35:189-95.

20. Pan K, Guan X, Li Y, Zhao J, Li J, Qiu H, et al. Clinical activity of adjuvant cytokine-induced killer cell immunotherapy in patients with postmastectomy triple-negative breast cancer. Clin Cancer Res. 2014;20:3003-11.

21. Chung M, Park J, Bang S, Park S, Song S. Phase II clinical trial of ex vivoexpanded cytokine-induced killer cells therapy in advanced pancreatic cancer. Cancer Immunol Immunother. 2014;63:939-46.

22. Wang M, Shi S, Qi J, Tang X, Tian J. S-1 plus CIK as second-line treatment for advanced pancreatic cancer. Med Oncol. 2013;30:747.

23. Plate JM, Plate AE, Shott S, Bograd S, Harris JE. Effect of gemcitabine on immune cells in subjects with adenocarcinoma of the pancreas. Cancer Immunol Immunother. 2005;54:915-25.

24. Nowak AK, Robinson BW, Lake RA. Gemcitabine exerts a selective effect on the humoral immune response: implications for combination chemoimmunotherapy. Cancer Res. 2002;62:2353-8.

25. Suzuki E, Sun J, Kapoor V, Jassar AS, Albelda SM. Gemcitabine has significant immunomodulatory activity in murine tumor models independent of its cytotoxic effects. Cancer Biol Ther. 2007;6:880-5.

26. Bauer C, Bauernfeind F, Sterzik A, Orban M, Schnurr M, Lehr HA, et al. Dendritic cell-based vaccination combined with gemcitabine increases survival in a murine pancreatic carcinoma model. Gut. 2007;56:1275-82.

27. Ghansah T, Vohra N, Kinney K, Weber A, Kodumudi K, Springett G, et al. Dendritic cell immunotherapy combined with gemcitabine chemotherapy enhances survival in a murine model of pancreatic carcinoma. Cancer Immunol Immunother. 2013;62:1083-91.

28. Khallouf H, Märten A, Serba S, Teichgräber V, Büchler MW, Jäger D, et al. 5Fluorouracil and interferon-alpha immunochemotherapy enhances immunogenicity of murine pancreatic cancer through upregulation of NKG2D ligands and MHC class I. J Immunother. 2012;35:245-53.

29. Schmidt-Wolf IG, Lefterova P, Mehta BA, Fernandez LP, Huhn D, Blume KG, et al. Phenotypic characterization and identification of effector cells involved in tumor cell recognition of cytokine-induced killer cells. Exp Hematol. 1993;21:1673-9.

30. Pievani A, Belussi C, Klein C, Rambaldi A, Golay J, Introna M. Enhanced killing of human B-cell lymphoma targets by combined use of cytokine-induced killer cell (CIK) cultures and anti-CD20 antibodies. Blood. 2011;117:510-8.

31. Nishimura R, Baker J, Beilhack A, Zeiser R, Olson JA, Sega El, et al. In vivo trafficking and survival of cytokine-induced killer cells resulting in minimal GVHD with retention of antitumor activity. Blood. 2008;112:2563-74.

32. Thorne SH, Negrin RS, Contag CH. Synergistic antitumor effects of immune cell-viral biotherapy. Science. 2006;311:1780-4.

33. Sangiolo D, Mesiano G, Gammaitoni L, Leuci V, Todorovic M, Giraudo L, et al. Cytokine-induced killer cells eradicate bone and soft-tissue sarcomas. Cancer Res. 2014;74:119-29.

34. Pievani A, Borleri G, Pende D, Moretta L, Rambaldi A, Golay J, et al. Dual-functional capability of CD3 + CD56+ CIK cells, a T-cell subset that acquires NK function and retains TCR-mediated specific cytotoxicity. Blood. 2011;118:3301-10.

35. Kornacker M, Moldenhauer G, Herbst M, Weilguni E, Tita-Nwa F, Harter C, et al. Cytokine-induced killer cells against autologous CLL: direct cytotoxic effects and induction of immune accessory molecules by interferon-gamma. Int J Cancer. 2006;119:1377-82

36. Hoffmann K, Mehrle S, Schmidt J, Büchler MW, Märten A. Interferonalpha restitutes the chemosensitivity in pancreatic cancer. Anticancer Res. 2008;28:1499-507.

37. Arina A, Bronte V. Myeloid-derived suppressor cell impact on endogenous and adoptively transferred T cells. Curr Opin Immunol. 2015;33C:120-5.

38. Vincent J, Mignot G, Chalmin F, Ladoire S, Bruchard M, Chevriaux A, et al. 5Fluorouracil selectively kills tumor-associated myeloid-derived suppressor cells resulting in enhanced $T$ cell-dependent antitumor immunity. Cancer Res. 2010;70:3052-61.
39. Le HK, Graham L, Cha E, Morales JK, Manjili MH, Bear HD. Gemcitabine directly inhibits myeloid derived suppressor cells in BALB/c mice bearing 4T1 mammary carcinoma and augments expansion of T cells from tumorbearing mice. Int Immunopharmacol. 2009;9:900-9.

40. Kanterman J, Sade-Feldman M, Biton M, Ish-Shalom E, Lasry A, Goldshtein A, et al. Adverse immunoregulatory effects of 5FU and CPT11 chemotherapy on myeloid-derived suppressor cells and colorectal cancer outcomes. Cancer Res. 2014;74:6022-35.

41. Huang A, Zhang B, Wang B, Zhang F, Fan KX, Guo YJ. Increased CD14(+)HLA-DR (-/low) myeloid-derived suppressor cells correlate with extrathoracic metastasis and poor response to chemotherapy in non-small cell lung cancer patients. Cancer Immunol Immunother. 2013;62:1439-51.

\section{Submit your next manuscript to BioMed Central and we will help you at every step:}

- We accept pre-submission inquiries

- Our selector tool helps you to find the most relevant journal

- We provide round the clock customer support

- Convenient online submission

- Thorough peer review

- Inclusion in PubMed and all major indexing services

- Maximum visibility for your research

Submit your manuscript at www.biomedcentral.com/submit
Biomed Central 